\title{
The Effectiveness of Platelet Rich Plasma Injection in Management of Rotator Cuff Tendinopathy
}

\author{
Wael Hamdi Farhat ${ }^{1, *}$ MSc; Ismail Ahmed Hammoda ${ }^{2}$ MD ; Emad Mohamed Zayed ${ }^{2}$ MD
}

\author{
*Corresponding Author: \\ Wael Hamdi Farhat \\ wael.hamdi2785@gmail.com
}

Received for publication November 13, 2020; Accepted january 5, 2021; Published online january 5, 2021.

Copyright 2020 The Authors published by Al-Azhar University, Faculty of Medicine, Cairo, Egypt. All rights reserved. This an openaccess article distributed under the legal terms, where it is permissible to download and share the work provided it is properly cited. The work cannot be changed in anyway or used commercially.

doi: 10.21608/aimj.2021.49733.1351

${ }^{1}$ Orthopedic Surgery Department, El Fayoum general hospital, Egypt

${ }^{2}$ Orthopedic Surgery Department, Faculty of Medicine, Al-Azhar University, Cairo , Egypt.

\begin{abstract}
Background: Degenerative changes and inflammation in the rotator cuff (RC) are the most important causes of shoulder pain. With early diagnosis and timely application of traditional non-surgical treatments, the prognosis is favorable. There is widespread interest in the use of PRP in the treatment of tendinopathy as well as an increasing number of randomized controlled trials studying the effectiveness of PRP in tendinopathy.

Aim of work: To assess the efficacy of platelet rich plasma injection in the treatment of rotator cuff tendinopathy.

Patient and Methods: From September 2018 till March 2020, twenty patients were treated by injection of single PRP in sub acromial space for chronic rotator cuff tendinopathy. Clinical examination and shoulder plain radiograph, and MRI were done for all patients. Pre injection and post injection visual analogue scale (VAS) and constant Murley score were obtained to assess pain and function.

Results: The visual analogue scale showed no significant improvement from 5.5 to 5.4 5.4 and constant Murley score from 66.9 to 68.8 .

Conclusion: Single PRP injection in the sub acromial space for rotator cuff tendinopathy therapy shows no significant improvement as regard reducing pain nor improving function.

Keywords: Rotator; cuff; tendinopathy; platelet rich plasma; sub acromial injection.
\end{abstract}

Disclosure: The authors have no financial interest to declare in relation to the content of this article. The Article Processing Charge was paid for by the authors.

Authorship: All authors have a substantial contribution to the article.

\section{INTRODUCTION}

The shoulder joint is one of the body's most flexible joints, this mobility that contributes to the high incidence of injury and degenerative pathology of the joint. ${ }^{1}$

Rotator cuff tendinopathy is one of the most common causes for shoulder pain however; the sub acromial bursa is a common cause of pain also and may contribute to the development of supraspinatus tendinopathy. 2

Tendinopathies and partial tears are often difficult to differentiate between, so care must be taken in the physical examination. ${ }^{3}$ Pain is the major presenting symptom of rotator cuff tendinopathy, Other signs may be weakness and/or pain on movement, particularly in overhead actions like reaching, throwing a ball, or swimming and also during rotation of the arm at the shoulder joint. ${ }^{4}$

MRI is the main noninvasive investigatory module used for diagnosis. 5
The ultimate goal of any therapeutic intervention for shoulder pain is the

restoration of pain-free function. ${ }^{6}$ Different modules of treatment are used such as:

physiotherapy $^{7}$, non-steroidal anti-inflammatory drugs (NSAIDs) ${ }^{6}$, nitric oxide (NO)

therapy $^{8}$, Hyperthermia ${ }^{9}$, Stem cell injection ${ }^{10}$, Corticosteroid injection $^{11}$ and platelet- rich plasma (PRP) injection. ${ }^{12}$

A blood derivative with a platelet concentration higher than that of entire blood, platelet-rich plasma (PRP), becomes a recent regenerative therapy to tissue damage and deterioration. The use of PRP in the management of tendinopathy and a growing number of randomized controlled trials (RCTs) examining the efficacy of PRP in the management of tendinopathy are of widespread interest. 13 
The present work aimed to assess the efficacy of platelet rich plasma injection in the treatment of rotator cuff tendinopathy.

\section{PATIENT AND MATERIALS}

This study was approved by the Ethical Committee of Al-Azhar University. From September 2018 till March 2020, 20 patients who underwent single PRP injection in sub- acromial space at Alazhar university hospitals were followed prospectively after 6 months (mean age, 44.9. age range, 20-61 years; 13 females and 7 males).

Inclusion criteria: Supraspinatus tendinopathy including small partial tear of supraspinatus tendon and patients not responding to NSAIDS and physiotherapy.

Exclusion criteria: Frozen shoulder, or severe shoulder arthritis, sub acromial impingement due to a bony cause, prior shoulder joint operation, rotator cuff tendon calcification and severe medical comorbidities such as malignant neoplasms, dyscrasia of the blood and severe infection.

All patients were examined generally and locally and shoulder plain radiograph, and MRI were done for all of them. Pre injection and post injection visual analogue scale (VAS) and constant Murley score were obtained to assess pain and function. Patient counseling was a crucial part of the procedure and written consent was acquired from every patient.

Preparation of Platelets rich plasma and injection technique: Under closed system blood withdrawal technique, $30 \mathrm{ml}$ volume of autologous blood became taken from every sufferer onto 4 vacutainer tubes, each one comprising $1 \mathrm{ml}$ anticoagulant citrate dextrose solution (ACD). In order to obtain the baseline entire blood platelets and leukocytes (WBC) value, an extra $2 \mathrm{~mL}$ of entire blood became collected into the ethylenediaminetetraacetic acid (EDTA) tube. PRP became prepared accordingly to a protocol for double-centrifugation.

First centrifugation: Using a laboratory centrifuge, separation of blood cell components has been carried out. The tubes became centrifuged for 6 mins at room temp at $1400 \mathrm{G}$, leading to two fundamental elements: the lower fraction blood cell component (BCC) and the upper fraction serum component (SEC).

Second centrifugation: Six $\mathrm{mm}$ under the line separating the BCC from the SEC, a mark was made. All contents over this point were pipetted to raise the overall quantity of platelets obtained for the second centrifugation and transfer without anticoagulant to one other $5 \mathrm{ml}$ vacutainer tube. The specimen had been then centrifuged once more for $2 \mathrm{~min}$ at $4000 \mathrm{G}$, leading to two components: SEC and PRP. The PRP was separated from the SEC (approximately $4 \mathrm{~cm}$ ). One $\mathrm{ml}$ sample was used for platelets count after the preparation of PRP. For best results, it should be 3-6 folds of platelets count of whole blood.
Activation of PRP was done immediately before injection by adding calcium chloride $0.1 \mathrm{ml}$ for every $1 \mathrm{ml}$ of PRP.

Injection technique: After Skin dis-infection with betadine, the patients were seated with his arm in the modified crass position. Identifying the spine of the scapula by palpation and follow it laterally to reach the acromion process. Using the postero- lateral approach a sterile needle of 5-ml. syringe was inserted percutaneously, 4 milliliters of PRP with concurrent usage of one $\mathrm{ml}$ of local anesthetic, was administered in the sub-acromial space.

Post -injection protocol: Acetaminophen was only allowed as an analgesic after injection.

The patients were advised to carry out a sluggish, un-resisted range of movement for 2 days after the injection. The sufferer started a band-resistant series of motion exercises in abduction, flexion, and internal and external rotation starting on the third day after the injection. Gradual return to activity.

Statistical Methods: Microsoft Excel software is used to codify, enter and analyse data collected through history, basic clinical evaluation, lab tests and result measures. Data was then imported into the analysis software Statistical Package for Social Sciences (SPSS version 20.0). Depending on the type of qualitative data, the quantitative continuous group represents a mean $\pm \mathrm{SD}$ as a number and percentage.

\section{RESULTS}

The mean score of VAS before injection was $(5.5 \pm 0.83)$ and mean pain was $(7.75 \pm 1.3)$, mean ADL was (15.1 \pm 1.8$)$, mean ROM was $(30.6 \pm 2.5)$, mean strength, and mean strength was $(13.45 \pm 2.4)$ mean total constant score was $(66.9 \pm 5.4)$. (Table 1).

\begin{tabular}{|c|c|c|c|}
\hline Variables & Mean & SD & Range \\
\hline \multicolumn{4}{|c|}{ Pre injection } \\
\hline VAS score & 5.5 & 0.83 & $4-7$ \\
\hline Pain & 7.75 & 1.3 & $5-10$ \\
\hline ADL & 15.1 & 1.8 & $12-18$ \\
\hline ROM & 30.6 & 2.5 & $26-34$ \\
\hline Strength & 13.45 & 2.4 & $10-18$ \\
\hline Constant & 66.9 & 5.4 & $59-78$ \\
\hline
\end{tabular}

Table 1: Description of VAS and constant score pre injection.

After 6 months follow up the mean VAS score was $(5.35 \pm 1.5)$ and mean pain was $(8.6 \pm 3.2)$, mean ADL was (15.2 \pm 3.1$)$, mean ROM was $(31 \pm 4.4)$, mean strength was (14 \pm 3.5$)$, and mean total constant score was (68.8 \pm 12.7$)$ (Table 2). 


\begin{tabular}{|c|c|c|c|}
\hline \multirow{4}{*}{ Variables } & Mean & SD & Range \\
\hline \multicolumn{4}{|c|}{ After 6 m } \\
\hline VAS score & 5.35 & 1 & $4-8$ \\
\hline Pain & 8.6 & 3 & $4-14$ \\
\hline ADL & 15.2 & 3 & $10-18$ \\
\hline ROM & 31 & 4 & $20-36$ \\
\hline Strength & 14 & 3 & $9-20$ \\
\hline Constant & 68.8 & 12.7 & $48-84$ \\
\hline
\end{tabular}

Table 2: Description of VAS and constant score after 6 months.

The results showed that there was no statistical significant improvement at end of treatment with $\mathrm{p}$ value $>0.05$ in VAS score follow up. Table (3)

\begin{tabular}{|c|c|c|c|c|}
\hline \multirow{2}{*}{ Follow up } & \multicolumn{2}{|c|}{ VAS score } & \multirow{2}{*}{$\begin{array}{c}\text { p- } \\
\text { value }\end{array}$} & Sig. \\
\cline { 2 - 3 } & Mean & SD & val & \\
\hline Pre injection & 5.5 & 0.83 & & \\
\hline After $6 \mathrm{~m}$ & 5.35 & 1.5 & 0.92 & NS \\
\hline
\end{tabular}

Table 3: Comparison of VAS score follow up among study group

In addition, there was no statistical significant improvement at end of treatment with p-value $>0.05$ in total constant score follow up. Table (4)

\begin{tabular}{|c|c|c|c|l|}
\hline \multirow{2}{*}{ Follow up } & \multicolumn{2}{|c|}{ Total constant score } & \multirow{2}{*}{ p- value } & \multirow{2}{*}{ Sig. } \\
\cline { 2 - 3 } & Mean & & \multirow{2}{*}{0.81} & NS \\
\hline Pre injection & 66.9 & 5.4 & 12.7 & \\
\hline After 6 m & 68.8 & & \\
\hline
\end{tabular}

Table 4: Comparison of total constant score follow up among study group

\section{DISCUSSION}

An important source of joint pain in the shoulder and a major cause of disabilities and work losses is rotator cuff tendinopathy (RCT). It becomes a common disease, with its incidence rising with age. ${ }^{2}$ There is a multifactorial origin of rotator cuff tendinopathy. Extrinsic elements, such as anatomical problems and changes in the kinematics of the joint or intrinsic elements, such as age-related degenerative alterations and vascular alterations, can play a function in the development of the RCT. The underlying mechanisms may differ between younger and older patients 14

Platelets rich plasma (PRP) is a plasma cellular component acquired through centrifugation of entire blood and has a better platelet concentration than entire blood. This involves different growth factors, which may play a position in the regeneration of tissues. 15

Despite several researches in the literary works investigating PRP management for the treatment of chronic tendinopathy, there is controversial proof thus far displaying the benefits of PRP injections.

An important source of joint pain in the shoulder and a major cause of disabilities and work losses is rotator cuff tendinopathy (RCT). It becomes a common disease, with its incidence rising with age. $^{2}$ There is a multifactorial origin of rotator cuff tendinopathy. Extrinsic elements, such as anatomical problems and changes in the kinematics of the joint or intrinsic elements, such as age-related degenerative alterations and vascular alterations, can play a function in the development of the RCT. The underlying mechanisms may differ between younger and older patients $^{14}$

Platelets rich plasma (PRP) is a plasma cellular component acquired through centrifugation of entire blood and has a better platelet concentration than entire blood. This involves different growth factors, which may play a position in the regeneration of tissues. ${ }^{15}$

Despite several researches in the literary works investigating PRP management for the treatment of chronic tendinopathy, there is controversial proof thus far displaying the benefits of PRP injections.

Further trials with a greater numbers of sufferers and longer follow-up times are required.

\section{CONCLUSION}

At 6 months follow-up, a single injection of PRP in sub acromial space for the treatment of RC tendinopathy showed no significant improvement at reducing pain or improving function.

\section{REFERENCES}

1. Factor D, Dale B. : Current Concepts of Rotator Cuff Tendinopathy.International journal of sports physical therapy; 2014;9(2):274.

2. Lewis JS : Rotator Cuff Tendinopathy: A Model for the Continuum of Pathology and Related Management. British Journal of Sports Medicine, 2010;44(13):918-23.

3. Hermans J, Luime JJ, Meuffels DE et al.: Does This Patient With Shoulder Pain Have Rotator Cuff Disease? The Rational Clinical Examination Systematic Review. Aug 28 2013; 310(8):837-47.

4. KELTON M. BURBANK, et al.: Chronic Shoulder Pain Part I: Evaluation and Diagnosis American Family Physician. Feb 15 2008; 77(4):453- 60. 
5. De Jesus JO, Parker L, Frangos AJ, Nazarian LN.: Accuracy of MRI, MR arthrography, and ultrasound in the diagnosis of rotator cuff tears: a meta-analysis. American Journal of Roentgenology : Jun 2009; 192(6):1701-7

6. Littlewood C, May S, Walters S. : A Review of Systematic Reviews of the Effectiveness of Conservative Interventions for Rotator Cuff Tendinopathy. SAGE : 2017; 5(3)

7. De Oliveira FCL, de Fontenay BP, Bouyer LJ, Desmeules F, Roy JS.: Effects of kinesiotaping added to a rehabilitation programme for patients with rotator cuff tendinopathy: protocol for a single-blind, randomised controlled trial addressing symptoms, functional limitations and underlying deficits. BMJ Open. 2017; 7(9):e017951

8. Murrell G. (2007): Using nitric oxide to treat tendinopathy. Br. J. Sports Med. 42: 227-231.

9. Giombini, A., Cesare, A., Safran, M., Ciatti, R., \&Maffulli, N.: Short- term effectiveness of hyperthermia for supraspinatus tendinopathy in athletes. American Journal of Sports Medicine, 2006; 34(8), 1247-52.

10. Ruzzini L, Longo UG, Rizzello G, et al.: Stem cells and tendinopathy: state of the art from the basic science to clinic application. Muscles Ligaments Tendons J. 2012; 2(3):235-8.

11. Ferhat Say, Deniz Gürler, Murat Bülbül. (): Platelet-Rich Plasma versus Steroid Injection for Subacromial Impingement Syndrome. Journal of Orthopaedic Surgery. 2016; 24(1):62-6.
12. Mautner $\mathrm{K}$ and Kneer L.: Treatment of tendinopathies with platelet- rich plasma. Phys Med Rehabil Clin N Am. 2014; 25:865-80.

13. Dhurat R and Sukesh M. : Principles and Methods of Preparation of Platelet-Rich Plasma: A Review and Author's Perspective. J Cutan Aesthet Surg. 2014; 7(4):189-97.

14. Seitz AL, McClure PW, Finucane S, Boardman ND, Michener LA. : Mechanisms of Rotator Cuff Tendinopathy: Intrinsic, Extrinsic, or Both? Clinical Biomechanics, Jan 31 2011; 26(1):1-2.

15. Paoloni J, De Vos RJ, Hamilton B, Murrell GA and Orchard J. : Platelet- rich plasma treatment for ligament and tendon injuries. Clin J Sport Med. 2011; 21:37-45.

16. Kesikburun S, Tan AK and Yilmaz B. : Platelet-Rich Plasma Injections in the Treatment of Chronic Rotator Cuff Tendinopathy: A Randomized Controlled Trial With 1-Year Follow-up, Am J Sports Med. 2013; 41: 2609-12.

17. Scarpone M, Rabago D, Snell E, et al. Effectiveness of Platelet-rich Plasma Injection for Rotator Cuff Tendinopathy: A Prospective Open-label Study. Glob Adv Health Med. Mar 2013;2(2):26-31

18. Shams, A., El-Sayed, M., Gamal, O. et al. : Sub acromial injection of autologous platelet-rich plasma versus corticosteroid for the treatment of symptomatic partial rotator cuff tears. Eur J Orthop SurgTraumatol . $2016 ; 26: 837$ 